\title{
Outcome of Sorafenib Therapy in Advanced Hepatocellular Carcinoma
}

\author{
Chowdhury MNG ${ }^{1}$, Islam MA ${ }^{2}$
}

DOI: https://doi.org/10.3329/jafmc.v15i1.48633

\begin{abstract}
Introduction: Hepatocellular carcinoma (HCC) is the most common type of primary liver cancer. It occurs most often in people with chronic liver diseases, such as cirrhosis caused by hepatitis B or hepatitis C infection. It is the fifth most common liver cancer globally and also a common cancer in Bangladesh. Treatment is difficult; however newer oral drug Sorafenib is available in our country which is promising.
\end{abstract}

Objectives: To evaluate the efficacy of TKI Sorafenib on advanced and non-resect able primary HCC in Bangladeshi patients.

Materials and Methods: This clinical trial was conducted on 38 diagnosed patients of advanced hepatocellular carcinoma (stage-3 and Child-Pugh Class-C) over a period of 3 years from May 2014 to July 2017 in the department of Oncology, CMH Dhaka. The patients receive Sorafenib $200-400 \mathrm{mg}$ twice daily till disease progression ceases or increases and there are no unacceptable toxicities. Efficacy was observed in terms of overall survival, progressive free survival (PFS), drug compliance and common toxicities.

Results: In 24 months of follow -up period from the date of Sorafenib taken, median overall survival was 7.73 months. The median overall survival was 8 months (range 8.13-27.43 months) in $42.4 \%$ patients. The median PFS is 3.5 months (range 1.2-5.8 months). Common toxicities found were anorexia, weakness, yellow colouration of skin and mucosa, HTN, rash and diarrhoea.

Conclusion: In Sorafenib treated Bangladeshi advanced HCC patients, the median overall survival was found to be significant and compatible with some of the international publication.

Key-words: Hepatocellular carcinoma, Sorafenib therapy, Outcome.

\section{Introduction}

Hepatocellular carcinoma (HCC) is the most common type of primary liver cancer. It occurs most often in people with chronic liver diseases, such as cirrhosis caused by hepatitis B or hepatitis $C$ infection. Hepatocellular carcinoma is the fifth most common cancer globally and also a common cancer in Bangladesh'. 5 year survivals $<10 \%$, incidence is rising. New era in the treatment of advanced HCC has been added in 2007 when Sorafenib got the FDA approval2. Sorafenib is a tyrosine protein Kinase inhibitor. Sorafenib Inhibit cell surface and intracellular kinases to reduce proliferation of Tumor cells. Generic Sorafenib became available in Bangladesh in the year 2012. The efficacy of Sorafenib is already established ${ }^{1}$. No data is available regarding efficacy of Sorafenib treatment for patients of advanced primary HCC in Bangladesh. To evaluate the efficacy of TKI Sorafenib on advanced and non resectable primary HCC (stage-3 and Child-Pugh Class-C) in patients of Bangladesh.

\section{Materials and Methods}

This clinical trial was conducted on 38 diagnosed patients of advanced hepatocellular carcinoma (stage-3 and Child-Pugh Class-C) over a period of 3 years from May 2014 to July 2017 in the department of Oncology, CMH Dhaka. Ethical approval obtained from CMH Dhaka. Median age of enrolled patients was 56 years (PS: 0-2). Informed written consent was taken from the patient's prior enrollment. The patients received Sorafenib 200-400 mg twice daily till disease progression ceases or increases and there are no unacceptable toxicities. Most of them received Sorafenib for $\mathrm{HCC}$ as first line therapy. The study carried out with patients having performance status ECOG (0-2). Efficacy was observed in terms of overall survival, progression free survival, drug compliance and common toxicities. Primary end point was overall survival. Data Analysis was done by SPSS 16.0.

\section{Results}

Total 38 patients mean age was found $58.4 \pm 17.1$ years with range from 30 to 93 years. Majority 35(92.1\%) patients were male, $37(97.4 \%$ ) were married, $30(78.9 \%)$ was found HBV related Chronic Liver Disease and 38(100\%) was found Child-Pugh Class C (Table-I). Median overall survival was found 7.73 months with range from 1.5 to 27.4 months. Median PFS was found 3.5months with range from 1.2 to 5.8 months (Table-II). Efficacy of Sorafenib found that $23(60.5 \%)$ patients was found in stable disease followed by $22(57.9 \%)$ in tumor response, $15(39.5 \%)$ in progressive disease and $5(13.2 \%)$ in partial response (Table-III). Complications of the study patients presented in Table-IV; all 38(100\%) patients had mucositis, 37(97.4\%) had thrush, 37(97.4\%) had palmoplanter syndrome, 15(39.5\%) had nausea, 14(36.8\%) had vomiting, 13(34.2\%) had fatigue and $12(31.6 \%)$ had abdominal pain.

Table-l: Demographic characteristics of the study patients $(n=38)$

\begin{tabular}{|c|c|c|c|}
\hline \multicolumn{2}{|c|}{ Demographic Characteristics } & Frequency & Percentage \\
\hline \multirow{6}{*}{ Age in years } & $<40$ & 4 & 10.5 \\
\hline & $41-50$ & 11 & 28.9 \\
\hline & $51-60$ & 10 & 26.3 \\
\hline & $61-70$ & 8 & 21.1 \\
\hline & $>70$ & 5 & 13.2 \\
\hline & \multicolumn{3}{|c|}{ Mean $\pm \mathrm{SD}=58.4 \pm 17.1 ;$ Range $=30-93$} \\
\hline \multirow{2}{*}{ Sex } & Male & 35 & 92.1 \\
\hline & Female & 3 & 7.9 \\
\hline \multirow{2}{*}{ Marital Status } & Married & 37 & 97.4 \\
\hline & Unmarried & 1 & 2.6 \\
\hline \multirow{3}{*}{ Cause of liver disease } & HBV & 30 & 78.9 \\
\hline & $\mathrm{HCB}$ & 4 & 10.5 \\
\hline & non-HBV+non-HCB & 4 & 10.5 \\
\hline Child-Pugh & Class $\mathrm{C}$ & 38 & 100 \\
\hline
\end{tabular}

1. Brig Gen Md Niamul Gani Chowdhury, MBBS, FCPS, Personal Physician to Hon'ble President of Bangladesh, President Office, Bangabhaban, Dhaka (E-mail: majniamul@yahoo.com) 2. Maj Gen Md Azizul Islam, MBBS, FCPS, Consultant Physician General, Bangladesh Armed Forces, Dhaka. 
Table-Il: Overall survival and PFS in the Bangladeshi population ( $n=38$ )

\begin{tabular}{|l|c|c|}
\hline \multicolumn{1}{|c|}{ Characteristics } & Median & Min-Max \\
\hline Overall survival (month) & 7.73 & $1.50-27.40$ \\
\hline PFS (month) & 3.50 & $1.20-5.80$ \\
\hline
\end{tabular}

Table-III: Therapeutic efficacy of Sorafenib $(n=38)$

\begin{tabular}{|l|c|c|}
\hline \multicolumn{1}{|c|}{ Efficacy of Sorafenib } & Frequency & Percentage \\
\hline Partial response & 5 & 13.2 \\
\hline Stable disease & 23 & 60.5 \\
\hline Progressive disease & 15 & 39.5 \\
\hline Tumor response & 22 & 57.9 \\
\hline
\end{tabular}

Table-IV: Complications of the study patients $(n=38)$

\begin{tabular}{|l|c|c|}
\hline \multicolumn{1}{|c|}{ Complications } & Frequency & Percentage \\
\hline Mucositis & 38 & 100 \\
\hline Oral thrush & 37 & 97.4 \\
\hline Plamoplanter syndrome & 37 & 97.4 \\
\hline Nausea & 15 & 39.5 \\
\hline Vomiting & 14 & 36.8 \\
\hline Fatigue & 13 & 34.2 \\
\hline Abdominal pain & 12 & 31.6 \\
\hline Vertigo & 10 & 26.3 \\
\hline Skin rash & 10 & 26.3 \\
\hline Hypertension & 9 & 23.7 \\
\hline Anorexia & 11 & 28.9 \\
\hline Diarrhoea & 5 & 13.2 \\
\hline Hyperbilirubinemia & 3 & 7.9 \\
\hline Rectal bleeding & 1 & 2.6 \\
\hline
\end{tabular}

\section{Discussion}

In this present study it was observed that mean age was found $58.4 \pm 17.1$ years with range from 30 to 93 years. Majority $35(92.1 \%)$ patients were male, $37(97.4 \%)$ were married, $30(78.9 \%)$ was found HBV positive and $38(100 \%)$ was in Child-Pugh class $\mathrm{C}$. In study of Abraham et al ${ }^{2}$ the male:female ratio for HCC in India is 4:1. Ji et $\mathrm{al}^{3}$ conducted an openlabel randomized study with 189 patients having advanced HCC Child-Pugh B or C. He divided HCC patients into two, groups, one with Sorafenib and other with best supportive care. Federico et al ${ }^{4}$ study observed that the median age was 69 years (range, 58-81 years) and the ratio of males to females was 18:8. Overall, 15 patients were infected with the hepatitis $\mathrm{C}$ virus (HCV), eight with $\mathrm{HBV}$ and three were co-infected with HCV/HBV. In total, 20(77\%) patients were presented with an underlying CP-A cirrhosis and 6(23\%) with CP-B. Parsons et $\mathrm{al}^{5}$. Study showed that the more than half $(54.0 \%)$ patients belonged to age over 60 years and male were $83.5 \%$. In advanced $\mathrm{HCC}$ patients, the majority had been diagnosed with chronic hepatitis $(52.6 \%)$, cirrhosis $(60.0 \%)$ and Child-Pugh score Class B (63.0\%). Song et al ${ }^{6}$ study observed that the median age for the patients was 56 years (range 21-81 years), and 32 of them $(80 \%)$ had HBV infection. Male-Female ratio was 33.7. Llovet et al ${ }^{1}$ study mean age was found $64.9 \pm 11.2$ Years, 260(87\%) was found hepatitis C virus infection and 284(95\%) was found in Child-Pugh class A.

In this current study, it was observed that median overall survival was found 7.73 months with range from 1.5 to 27.4 months. Abraham et $\mathrm{al}^{2}$ observed median overall survival was 3.8 months, ranging from 12 to 885 days after Sorafenib therapy. Ji et al ${ }^{3}$ reported that median overall survival was 4 months and 3.5 months in the Sorafenib group and best supportive care group respectively. Kostner et al ${ }^{7}$ conducted a retrospective study with 76 patients in 2011 in Denmark found that patients in performance status 0-1 had a median overall survival of 6.2 months compared to 1.8 months in patients with poorer performance status. ChildPugh A patients had median overall survival of 6.6 months versus 3.6 months among patients with Child-Pugh B or C. Federico et $\mathrm{al}^{4}$ the median OS was $7.4(95 \% \mathrm{Cl}, 3.2-11.6)$ months. L lovetetal ${ }^{1}$ conducted median survival 10.7 months in the Sorafenib group. In the study of Song T et al ${ }^{6}$, the median OS was 12.7 months. In this study, it was observed that $23(60.5 \%)$ patients were found in stable disease followed by $22(57.9 \%)$ in tumor response, $15(39.5 \%)$ in progressive disease and $5(13.2 \%)$ in partial response. Federico et al ${ }^{4}$ observed that a partial response was observed in three patients $(12 \%)$, stable disease lasting at least 12 weeks was observed in 13 patients (50\%) and progression of disease was observed in 10 patients (38\%). Song et al ${ }^{6}$ study showed that five patients (12.5\%) achieved a partial response and 24 patients $(60 \%)$ achieved stable with a disease control rate of $60 \%$.

In this Series, it was observed that all $(100.0 \%)$ patients had mucositis, 37(97.4\%) had oral thrush, 37(97.4\%) had plamoplanter syndrome, 15(39.5) had nausea, 14(36.8\%) had vomiting, $13(34.2 \%)$ had fatigue and $12(31.6 \%)$ had abdominal pain. Abraham et $\mathrm{al}^{2}$ study reported that the adverse drug reaction documented in this study was vomiting, abdominal pain, fatigue, anorexia, hyperbilirubinaemia, diarrhoea, hand-foot syndrome, skin rash Rectal bleeding, insomnia, constipation, thrombocytopenia and abdominal discomfort. Kostner et $\mathrm{al}^{7}$ found that fatigue $(68 \%)$ was the main adverse drug reaction followed by anorexia (47\%), diarrhea $(42 \%)$, rash $(33 \%)$, nausea $(32 \%)$ and hand-foot syndrome (HFS) (28\%). Sorafenib is generally tolerable also in more compromised patients as severity of adverse events do not differ significantly between the patients with good versus poor performance status and liver function. Llovet JM et al ${ }^{1}$ reported that in Sorafenib group all 38 patients had diarrhea, 22 where fatigue, 21 had hand-foot skin reaction and 16 had rash or desquamation. Federico et al ${ }^{4}$ reveled the most common G3 toxicities were hand-foot-skin (HFS) reaction $(23 \%)$, malaise $(15 \%)$, diarrhoea $(7 \%)$ and mucositis $(4 \%)$. Song et al ${ }^{6}$ in Sorafenib treated patients are summarized, with HFS and diarrhoea as the most prevalent at $52.5 \%$ and $52.5 \%$, respectively. All of the patients who had HFS were presented with diarrhoea. Fatigue was found in seven patients (17.5\%) and hypertension in four patients $(10.0 \%)$.

According to this study, Sorafenib is recommended first line drug for the treatment of advanced and stage- 3 and Child-Pugh Class-C, HCC. It is observed that some of the patients with HCC 
respond quite well to Sorafenib but others do not respond equally. There might have some specific gene mutation like EGFR, ALK, BRAF, HER-2, KRAS etc which could be more sensitive to Sorafenib or any other TKI ${ }^{7}$. So detection of the unknown gene mutation in HCC is recommended which may further have improvedthe survival and prognosis of HCC.

\section{Conclusion}

In advanced HCC patients in the study, who have been treated with Sorafenib the median overall survival was found to be significant and compatible with the international publications. A further prospective study need to be conducted to get more comprehensive data and result.

\section{References}

1. Llovet JM, Ricci S, Mazzaferro V et al. Sorafenib in Advanced Hepatocellular Carcinoma. New England Journal of Medicine 2008; 359(4):378-90.
2. Abraham A, Purushothaman C, Damien D et al. Efficacy of Sorafenib Therapy in Patients with Advanced Hepatocellular Carcinoma in Indian Population. Hepatoma Res 2016; 2:224-8.

3. JiYX, Zhang ZF, Lan KT et al. Sorafenib in liver function impaired advanced hepatocellular carcinoma. Chin Med Sci J 2014; 29:7-14.

4. Federico A, Orditura M, Cotticelli $G$ et al. Safety and efficacy of Sorafenib in patients with advanced hepatocellular carcinoma and ChildPugh A or B Cirrhosis. Oncol Lett 2015; 9(4):1628-32.

5. Parsons HM, Chu Q, KarlitzJJ et al. Adoption ofSorafenib for the Treatment of Advanced-Stage Hepatocellular Carcinoma in Oncology Practices in the United States. Liver Cancer 2017; 6:216-26.

6. Song T, Zhang W, Wu $Q$ et al. A single center experience of Sorafenib in advanced hepatocellular Carcinoma patients: evaluation of prognostic factors. Eur J Gastroenterol Hepatol 2011; 23(12):1233-8.

7. Kostner AH, Sorensen M, Olesen RK et al. Sorafenib in advanced hepatocellular carcinoma: A nationwide retrospective study of efficacy and tolerability. Scientific World Journal 2013; 2013:931-72. 